ISSN: 2238-8052

\title{
COOPERAZIONE DECENTRATA TRA LA REGIONE EMILIA-ROMAGNA E STATO DEL PARANÀ PER LO SVILUPPO DEL COOPERATIVISMO E DELLE FILIERE AGROALIMENTARI DI QUALITÀ: IL CASO DEL PROGRAMMA BRASIL PRÓXIMO
}

\author{
COOPERAÇÃO DESCENTRALIZADA ENTRE A REGIÃO EMILIA-ROMAGNA E O ESTADO DO \\ PARANÁ PARA O DESENVOLVIMENTO DO COOPERATIVISMO E DAS CADEIAS \\ AGROALIMENTARES DE QUALIDADE: O CASO DO PROGRAMA BRASIL PRÓXIMO
}

Silvia GRANDI ${ }^{1}$

Artigo recebido em 30/09/2017 e aceito em 27/11/2017

Palavras-chave:

Cooperação internacional

Cadeias agroalimentares Territórios Desenvolvimento

Parole chiavi: Cooperazione internazionale Filiere agroalimentari Territori Sviluppo

\section{R E S U M O}

A cooperação entre as áreas subnacionais, comumente denominada cooperação descentralizada ou, mais recentemente, "parceria territorial" de acordo com a nova lei italiana sobre cooperação para o desenvolvimento (Lei 125/14), geralmente assume um papel marginal em termos financeiros, mas pode ser muito relevante em termos de eficácia e influência nas políticas de desenvolvimento local. É o que emerge do programa Brasil Próximo, no qual cinco regiões italianas (Umbria, Marche, Toscana, EmiliaRomagna, Ligúria) criaram entre 2004 e 2015 um sistema articulado de relações e planejamento para cooperação de transição post-aid. 0 objetivo foi alcançado através da ativação de uma ampla rede, o fortalecimento e o desenvolvimento de políticas e instrumentos, a criação de oportunidades recíprocas - incluindo a comercial - e as intervenções destinadas a apoiar processos endógenos de desenvolvimento sustentável local capazes de resolver os problemas socioeconômicos relativos a uma distribuição desequilibrada da riqueza. Em particular, este artigo chama a atenção para o relacionamento da Emilia-Romagna com o Estado do Paraná, analisando as atividades empreendidas para fortalecer políticas e projetos-piloto visando apoiar pequenos produtores nas cadeias agro-alimentares através do crescimento de microempresas, PME, o sistema de feira comercial local e o cooperativismo. Trata-se de uma abordagem basicamente baseada em pessoas, compartilhando boas práticas na região e nas redes. Os dilemas sempre permanecem, após a conclusão de projetos de cooperação bemsucedidos, representados pelas seguintes questões: A ação foi realmente sustentável? 0 que significa ser sustentável para as partes envolvidas? Como a política afeta a sustentabilidade desses processos?

\section{RIASSUNTO}

La cooperazione tra aree subnazionali, comunemente chiamata cooperazione decentrata o più recentemente "partenariato territoriale" nella nuova legge italiana per la cooperazione allo sviluppo (L. 125/14), assume di solito un ruolo marginale in termini finanziari ma può risultare molto rilevante in termini di efficacia ed influenza nelle policy per lo sviluppo locale. È quanto emerge del programma Brasil Próximo in cui cinque Regioni italiane (Umbria, Marche, Toscana, Emilia-Romagna, Liguria) hanno creato, tra il 2004 e il 2015, un articolato sistema di relazione e di progettualità in un'ottica di cooperazione di transizione post-aid. L'obiettivo si è concretizzato con l'attivazione di un vasto network, di rafforzamento e sviluppo di politiche e strumenti, di

\footnotetext{
${ }^{1}$ Università degli Studi di Modena e Reggio Emilia (Italia). E-mail: silvia.grandi@unimore.it
} 
creazione di reciproche opportunità - anche commerciali - e di interventi tesi ad accompagnare processi endogeni di sviluppo locale sostenibili capaci di intervenire sui problemi socio-economici derivanti da una squilibrata distribuzione della ricchezza. In particolare questo paper pone l'attenzione sul rapporto della Regione Emilia-Romagna con lo Stato del Paranà analizzando le attività svolte per il rafforzamento di politiche e di progetti pilota volti a sostenere i piccoli produttori nelle filiere agroalimentari attraverso la crescita delle microimprese, delle PMI, del sistema fieristico locale specialistico e del cooperativismo. Un approccio sostanzialmente basato sulle persone, sulla condivisione delle buone prassi maturate nel territorio regionale e sulla mise en reseau. I dilemmi sempre aperti dopo la conclusione anche dei progetti di cooperazione considerati di successo sono: è stato veramente sostenibile? Cosa significa sostenibile per le parti in gioco? Quanto la politica influisce nella sostenibilità di questi processi?

\section{LA COOPERAZIONE TRA AREE SUBNAZIONALI: INTRODUZIONE ED APPROCCIO METODOLOGICO}

La cooperazione tra aree subnazionali di due diversi Paesi è comunemente chiamata cooperazione decentrata, o più recentemente "partenariato territoriale", come si legge nella nuova normativa italiana per la cooperazione allo sviluppo (L. 125/2014). In essa, tuttavia, sembra emergere la volontà di sottolinearne i limiti e la subordinazione alla competenza esclusiva statale2, piuttosto che rafforzarne uno spazio di azione o riconoscerne il valore. È un atteggiamento del legislatore che può essere ricondotto ad alcune esperienze che, negli anni, hanno creato un acceso dibattito tra il Ministero degli affari esteri e della cooperazione internazionale italiano, da una parte, ed il sistema delle Autonomie locali, dall'altra. Queste ultime, in base a quanto emerge dalle interviste effettuate, hanno utilizzato lo strumento con troppa autonomia rispetto alle percezioni del Ministero, mancando di informazione, coordinamento e condivisione con il governo centrale. Inoltre, si desume dalle interviste che le Organizzazioni Non Governative hanno maggiore visibilità e riconoscimento nei processi e nelle progettualità di cooperazione mettendo in secondo piano le competenze potenziali delle istituzioni.

In effetti, il meccanismo di governance, potenzialmente multilivello, e l'applicazione del principio di sussidiarietà portano ad un sistema complesso di relazioni, organizzazioni, strumenti di

\footnotetext{
2 All'art. 9 della legge n. 125 dell'11 agosto del 2014 si legge: "I rapporti internazionali delle regioni e delle province autonome di Trento e di Bolzano, relativi alla cooperazione allo sviluppo, si svolgono nel rispetto dei princìpi contenuti nella presente legge o in altre leggi dello Stato o da esse desumibili, nonché nel rispetto della competenza esclusiva statale in materia di politica estera e di rapporti internazionali dello Stato, di cui all'articolo 117, secondo comma, lettera a) , della Costituzione. Ai fini dell'adozione delle leggi delle regioni e delle province autonome di Trento e di Bolzano volte a disciplinare le iniziative di cooperazione e di solidarietà internazionale sulla base della loro potestà legislativa, le disposizioni della presente legge sono princìpi fondamentali. Resta fermo quanto previsto dalla legge 5 giugno 2003, n. 131. 2. Le regioni e le province autonome di Trento e di Bolzano e gli enti locali possono attuare iniziative di cooperazione allo sviluppo, previo parere favorevole del Comitato congiunto di cui all'articolo 21 e nei limiti di quanto disposto dal comma 1 del presente articolo, di norma avvalendosi dell'Agenzia di cui all'articolo 17. Le regioni, le province autonome e gli enti locali comunicano preventivamente al Ministro degli affari esteri e della cooperazione internazionale e all'Agenzia di cui all'articolo 17 le attività di partenariato territoriale, finanziate e programmate, ai fini dell'applicazione dell'articolo 11 , commi 1 e 2 , e dell'inclusione delle attività stesse nella banca dati di cui all'articolo 17, comma 9".
} 
polity e policy. Ciò vede il coinvolgimento di una pluralità di soggetti: governo centrale, governi regionali, governi locali, organizzazioni non governative, associazionismo e terzo settore, sistema sindacale, sistema educativo e della ricerca e sempre più anche il settore imprenditoriale3.

D’altra parte, se si adotta un approccio territorialista, la cooperazione decentrata trova il suo fondamento teorico nel paradigma dello sviluppo locale, per quanto in pratica variamente interpretato (Dansero et al., 2008). Senza entrare nel dibattito sul complesso rapporto tra lo sviluppo locale e la cooperazione allo sviluppo, per il quale si rimanda alla letteratura (Boggio e Dematteis, 2002; Dansero et al., 2008; Bignante et al., 2008; Boggio et al., 2008; UNIDO, 2010; Grandi, 2010; Grandi e Curiazi, 2013; Dansero e Scarpocchi, 2014; Bignante et al., 2015; Bertoccin et al., 2016; Bini, 2016), l'approccio che idealmente accompagna la cooperazione decentrata è tipicamente bottom-up e peer-to-peer. L'azione di cooperazione e di sviluppo ha una maggiore probabilità che sia sostenibile, quindi anche duratura nel tempo, se la progettazione parte dalle esigenze locali; questa deve essere portata avanti attraverso la partecipazione e l'integrazione delle competenze locali di due o più aree subnazionali paritetiche: regione-regione, città-città o altre forme di comunità della società riferite ad una scala locale (Dansero et al., 2008; Grandi e Curiazi, 2013).

Lo sviluppo locale diventa la chiave metodologica principale nel dialogo tra i territori che, attraverso la cooperazione decentrata, cercano di trovare scambi di informazioni, idee, tecnologie, beni, know-how, ecc. volti ad auspicare, ispirare e, possibilmente, attuare progetti che per tutte le parti portino a "qualcosa di maggiormente desiderabile nel tempo di riferimento" e nei territori, ossia "sviluppo" (Grandi, 2013, p. 9). Nascono così partenariati fondati sulla visione di co-sviluppo tra territori.

In altri termini l'OICS - Osservatorio Interregionale Cooperazione Sviluppo (2005) -ha definito la cooperazione decentrata "un approccio alla cooperazione internazionale basato su modalità di partenariato territoriale, imperniate su accordi-quadro tra territori e comunità locali del nord e del sud del mondo che cooperano coinvolgendo in un impegno organico e continuo tutti gli attori delle rispettive realtà" per far crescere le conoscenze, le capacità gestionali e manageriali; trasferire e confrontare competenze e pratiche, per affrontare in maniera innovativa i problemi dello sviluppo, della qualità della vita, per un ambiente ed una economia sostenibile.

\footnotetext{
${ }^{3}$ All'art. 9 "Sistema della cooperazione italiana allo sviluppo" della legge n. 125 dell'11 agosto del 2014 si legge: "1. La Repubblica riconosce e promuove il sistema della cooperazione italiana allo sviluppo, costituito da soggetti pubblici e privati, per la realizzazione dei programmi e dei progetti di cooperazione allo sviluppo, sulla base del principio di sussidiarietà. 2 . Sono soggetti del sistema della cooperazione allo sviluppo:

a) le amministrazioni dello Stato, le università e gli enti pubblici;

b) le regioni, le province autonome di Trento e di Bolzano e gli enti locali;

c) le organizzazioni della società civile e gli altri soggetti senza finalità di lucro di cui all'articolo 26;

d) i soggetti con finalità di lucro, qualora agiscano con modalità conformi ai princìpi della presente legge, aderiscano agli standard comunemente adottati sulla responsabilità sociale e alle clausole ambientali, nonché rispettino le norme sui diritti umani per gli investimenti internazionali".
} 
Nel quadro delle forme di cooperazione internazionale allo sviluppo, la cooperazione decentrata è tra le più recenti in termini di riconoscimento strutturato a scala normativa, tuttavia, riferendosi nel presente articolo a quello tra regioni italiane e brasiliane, le radici si possono ricondurre a tre processi più o meno coevi. Questi sono nati negli anni cinquanta del Novecento, si sono nel tempo contaminati ed hanno trovato una sintesi nel partenariato territoriale attraverso:

- la cooperazione internazionale allo sviluppo;

- $\quad$ i gemellaggi tra città;

- i movimenti legati alle diaspore e alle catene migratorie nel mondo di natura politica ed economica4.

Per quanto riguarda la relazione con il gemellaggio tra città, particolarmente evocativa è la definizione classica usata da Jean Bareth, uno dei fondatori del Consiglio dei Comuni e delle Regioni d'Europa (CCRE), dopo la seconda guerra mondiale: "un gemellaggio è l'unione di due comunità che, in tal modo, tentano di agire partendo da una prospettiva europea e con l'obiettivo di affrontare i loro problemi e di instaurare tra loro legami sempre più stretti di amicizia" (CCRE, 2017)5. Questa evidenzia l'importanza del rapporto paritetico tra luoghi come determinante per migliorare la consapevolezza e la progettualità per risolvere "problemi". Ricordando Vallega (1995, pp. 79-80), un sistema locale nel suo processo autopoietico, quando assume la consapevolezza della sua capacità auto-organizzatrice tenderà, nel reagire agli stimoli esterni - come appunto una relazione di cooperazione e/o gemellaggio - ad avere come riferimento un suo progetto a cui riconosce un valore identitario. Nel reagire agli stimoli esterni forniti nella relazione con altri pari, compie operazioni concatenate con altre che avvengono al suo interno, tese a mantenere un livello di organizzazione appropriato per consentire il raggiungimento delle finalità predefinite in modo equilibrato.

Negli anni sono nate, si sono sviluppate (ed a volte si sono affievolite o concluse) relazioni tra luoghi fisicamente lontani fra di loro, ma che riconoscono punti in comune, ad esempio nelle loro storie, nelle loro origini o nelle similitudini dei sistemi di valori politici o economici, dai quali partire per realizzare iniziative di scambio culturale, economico e politico fra componenti delle due realtà o iniziative comuni. A livello europeo, con l'art. 20 delle disposizioni generali della Quarta Convenzione di Lomé firmata nel 1989, si affermò il principio di una cooperazione decentrata realizzata attraverso il concorso di parti attive economiche, sociali e culturali tra cui i poteri pubblici decentrati. Nel 1992, quest'approccio è stato esteso ai paesi in via di sviluppo dell'America Latina e dell'Asia. Le conferenze

\footnotetext{
${ }^{4}$ A quest'ultimo sistema di relazione andrebbero aggiunte le spinte legate allo sviluppo di interessi e relazioni commerciali e per l'internazionalizzazione o come ultimamente viene identificata "economic diplomacy", che proprio per la natura spesso localizzata dei sistemi produttivi ricerca sistemi produttivi locali omologhi o complementari esistenti o con fattori localizzativi adatti all'attrazione degli investimenti.

5 Parallelamente Eisenhower, negli Stati Uniti nel 1956 supportò il programma di sister cities all'interno della National League of Cities volto ad una cooperazione people-to-people per la costruzione di legami duraturi di pace (Sister Cities International, 2006). Riferimento interessante inoltre è lo studio UN-Habitat e UTO/FMCU (2001) che evidenzia la cooperazione decentrata come rapporti di cooperazione tra città, city-to-city, nell'ambito di uno sviluppo sostenibile e nei processi Habitat dell'ONU.
} 
delle Nazioni unite (Rio de Janeiro 1992, Istanbul 1996) sull'ambiente, sullo sviluppo e sugli insediamenti umani, il Vertice del millennio del 2000 e il Vertice mondiale di Johannesburg sullo sviluppo sostenibile hanno poi formalmente riconosciuto il loro ruolo nonché a scala europea, più recentemente, sono stati particolarmente rilevanti la Comunicazione "Le autorità locali: attori di sviluppo" (Commissione Europea, 2008) e le attività dell'Assise della Cooperazione Decentrata promossa dal Comitato delle Regioni.

In un terreno fertile, alla luce delle varie esperienze e relazioni internazionali delle città e di altri enti locali, nel 1991, per volontà della Conferenza delle Regioni e delle Province autonome italiane nacque l'OICS, l'osservatorio interregionale per la cooperazione allo sviluppo, una struttura comune in materia di cooperazione decentrata allo sviluppo e di sostegno ai processi di internazionalizzazione economica territoriale.

L'azione delle Regioni si è quindi intensificata mettendo a disposizione risorse di bilancio e sviluppando progetti svolti dalle Autonomie locali italiane, singolarmente o in consorzio fra loro. In questo caso si osserva anche il concorso delle espressioni della società civile organizzata attiva nel territorio di relativa competenza amministrativa e l'attuazione delle attività in partenariato prioritariamente con omologhe istituzioni dei PVS. In questo modo viene favorita la partecipazione attiva delle diverse componenti della società civile dei paesi partner nei processi decisionali finalizzato allo sviluppo sostenibile del loro territorio (MAECI-DGCS, 2002).

L'esperienza storica della cooperazione decentrata italiana oltre ad essere stata fortemente legata alla gestione di situazioni emergenziali e agli interventi umanitari ed in campo medico, ha visto l'attuazione di accordi e progetti volti a:

- appoggiare "tra pari" i processi di decentramento, politico e amministrativo per facilitare una maggiore democratizzazione attraverso il rafforzamento delle istituzioni a livello locale con scambi e rapporti tra i politici eletti delle due comunità e sostenere la cooperazione tecnica tra gli amministratori pubblici;

- creare ambienti favorevoli alla crescita di imprenditorialità per la creazione di lavoro attraverso forme associative di tipo cooperativistico e di micro, piccole e medie imprese (PMI), meccanismi creditizi equi e sostenibili;

- $\quad$ sviluppare centri di formazione professionale e specialistica - incluso quella universitaria e quindi per la ricerca scientifica e tecnologica -per la crescita qualitativa del saper fare, creare, produrre e vendere;

- creare spazi ed occasioni per il confronto culturale, sportivo e artistico nelle varie forme.

In termini finanziari, rispetto alla cooperazione internazionale allo sviluppo, quella decentrata rappresenta ancora una parte non particolarmente rilevante. 
La mappatura delle iniziative è assai complessa in quanto gli attori regionali o locali hanno un ruolo di donatori, quando stanziano risorse proprie che eventualmente vengono messe a disposizione mediante bandi o che vengono gestite direttamente6. Invece diventano organizzazioni finanziate a loro volta dalle istituzioni sovraordinate quando partecipano a bandi, ad esempio, del Ministero degli affari esteri e della cooperazione internazionale, dell'Agenzia per la Cooperazione Internazionale, della Commissione Europea7 o delle agenzie multilaterali.

La riflessione di questo contributo, dopo questa prima sintesi generale sullo sviluppo e sulle basi della cooperazione decentrata, pone l'attenzione sul rapporto della Regione Emilia-Romagna con lo Stato del Paranà analizzando le attività svolte per il rafforzamento di politiche e di progetti pilota volti a sostenere i piccoli produttori nelle filiere agroalimentari attraverso la crescita delle microimprese, delle PMI, del sistema fieristico locale specialistico e del cooperativismo. Un approccio sostanzialmente basato sulle persone, sulla condivisione delle buone prassi maturate nel territorio regionale e sulla mise en reseau. I dilemmi sempre aperti dopo la conclusione anche dei progetti di cooperazione considerati di successo sono: è stato veramente sostenibile? Cosa significa sostenibile per le parti in gioco? Quanto la politica influisce nella sostenibilità di questi processi? Quindi quest'analisi di caso porta ad alcune conclusioni sull'“inafferrabile” (Easterly, 2002) ruolo dei territori nello sviluppo.

Per quanto riguarda i metodi di indagine utilizzati, accanto alla revisione critica della documentazione istituzionale, politica e giornalistica, si è potuto usare l'approccio di ricerca etnografico, osservando direttamente i processi di decisione, gestione e governance durante le riunioni ed eventi nonché intervistando osservatori privilegiati

\section{LA COOPERAZIONE DECENTRATA TRA LA REGIONE EMILIA-ROMAGNA ED IL BRASILE: IL CASO DEL PROGRAMMA BRASIL PRÓXIMO}

Le tracce della cooperazione decentrata della Regione Emilia-Romagna si fondano su una feconda relazione sindacale e politica di cooperazione tra le organizzazioni sindacali e le scuole politiche attive da metà degli anni ottanta con l'effetto di diffondere i modelli emiliano-romagnoli ed italiani del cooperativismo, le politiche industriali e dei lavoratori. Istituzionalmente nel 1998 fu firmato un primo protocollo d'intesa sottoscritto tra la Regione Emilia-Romagna e lo stato di San Paolo

\footnotetext{
${ }^{6}$ In base ai dati ufficiali, le risorse in gioco rappresentano tuttavia solo una parte minore delle risorse relative alla cooperazione internazionale in Italia; nel 2000 infatti su un volume di 400 milioni di euro, solo il 12,5\% era in effetti costituito da risorse locali proprie, le altre - pur dirottate in questo tipo di cooperazione - erano derivanti da fondi nazionali, europei o internazionali.

7 L'Unione Europea ha alcuni programmi dedicati in cui anche Regioni, Comuni, ecc. sono eleggibili al finanziamento. Pur lungi dall'essere esaustivo, dal 2007 al 2012 grazie al programma NSL-LA (Attori non statali - Autorità locali) sono stati finanziati 480 progetti realizzati da autorità locali e regionali dell'Unione con partner dei paesi terzi, per un impegno di 171,7 milioni di euro (Platforma, 2013).
} 
(Pareschi e Breveglieri, 2013) mentre nel 1999 fu cofinanziato con fondi regionali un primo progetto coordinato da organizzazioni sindacali e socio-religiose dell'area di Rio de Janeiro e fu dedicato alla realizzazione di un Osservatorio sullo sfruttamento minorile in Brasile (Fanini e Preus, 2001). Dal 2001 al 2010 poi cominciarono ad essere finanziati annualmente, fino a quasi una decina all'anno, progetti volti, oltre che a supporto di politiche sociali, sempre più anche allo sviluppo di iniziative di cooperativismo, economia solidale e rurale, con particolare attenzione a quella agroalimentare.

Dopo le prime attività di collaborazione con l'area di Rio de Janeiro, ben presto l'attività progettuale si è diffusa in molte altre aree sia a scala di quartiere metropolitano, sia di aree rurali che di stati: Amazonas, Piauì, Parà, Recife, Sao Bernardo do Campo, San Paolo, Rio Grande Do Soul, Santa Catarina, Paranà, Salvador Bahia, ecc. D’altra parte anche il partenariato dei progetti diventò più articolato e, oltre alle organizzazioni sindacali, cominciarono ad essere coinvolte amministrazioni comunali, università e cooperative anche di significative dimensioni.

Tabella 1: Progetti Cofinanziati dalla Regione Emilia-Romagna 1999-2010.

Titolo del progetto

Realizzazione di un Osservatorio sullo sfruttamento minorile in Brasile
Anno di finanziamento

Prevenzione e reinserimento sociale dei meninos da rua nella municipalità di Ananindeua

Formazione ed avvio al lavoro di giovani donne a rischio - Nova Iguaçu (Rio de Janeiro)

Progetto Novo Marotinho - Intervento di assistenza socio-economica e formazione

Formazione Professionale e lotta alla disoccupazione per giovani e donne delle favelas di Sao

Bernardo do Campo Brasile

Sostegno e valorizzazione di giovani donne a rischio

Accoglienza formazione lavoro per ragazzi ciechi nella Bahia

Sviluppo di arte e cultura in 10 insediamenti della Riforma Agraria in Brasile. In collaborazione con il Movimento dei Sem Terra

Ristrutturazione coperto e cucina Allan Kardec - Alr Ceci CostaIl

Lo sviluppo del movimento cooperativo, alternativa concreta per i lavoratori

Cammini per le donne: Organizzazione di Reti Solidali di Produzione

Arte di educare 2

Prevenzione dell'abbandono, accoglienza e reinserimento familiare di bambini in situazione di rischio

Economia solidale speranza per il nord-est brasiliano

Imprenditoria solidale contro la povertà nelle favelas a Sao Bernardo do Campo Brasile

Bilancio partecipativo; formazione per consiglieri comunitari e membri del COMFORÇA

Sostegno al programma fame zero; cisterne e agricoltura familiare

Sviluppo della filiera produttiva lattiero casearia dell'Alto Acre

Lo sviluppo del movimento cooperativo: una prospettiva concreta per i lavoratori e le lavoratrici

Iscos ER

Progetto Di Cooperative Di Donne In Rete - Belo Horizonte, San Paolo, Recife, Nova Uguacu

Economia Solidale Speranza per il Nord-Est Brasiliano - Secondo Stralcio

Progetto di Sviluppo Cooperativo; una Prospettiva Concreta per i Lavoratori E Le Lavoratrici

Del Brasile- Abc Paulista (Stato Di San Paolo, Stato Di Rio Grande Do Soul, Santa Catarina,
1999

2001

2001

2001

2002

2002

2002

2002

2002

2002

2002

2003

2003

2003

2003

2003

2003

2003

2003

2003

2004

2004

2004 
Paranà)

Sostegno al Programma Fame Zero - Cisterne e Agricoltura Familiare nello Stato Del Piaù̀

Progetto di "Sviluppo della Filiera Produttiva Lattiero-Casearia dell'Alto Acre" - Rio Branco -

Acre

Progetto di Recupero ed Educazione Ambientale negli Ecosistemi di Foresta Atlantica della

Valle del Rio Dôce - Minas Gerais

Progetto di Imprenditoria Solidale Contro La Povertà - Municipio Di Sao Bernardo Do Campo

Progetto "Scuole della Partecipazione" Politiche Pubbliche e Partecipazione Popolare - Belo

Horizonte

"Sabor natural do sertao" appoggio alla commercializzazione dei prodotti dell'agricoltura

familiare nel semiarido nordest

Imprenditoria ed economia solidale nel grande abc di San Paolo

Promozione dei temi dell'economia solidale come strumento di inclusione sociale nelle aree

disagiate di Rio de Janeiro

Sostegno al Programma "Fame Zero", sostegno all'agricoltura familiare, centro polivalente di

Papera

Sostegno al rafforzamento delle cooperative popolari urbane della periferia di Salvador

Promozione di centri per favorire l'inserimento lavorativo di giovani e sviluppo del

movimento cooperativo in Brasile

Promozione dei temi dell'economia solidale come strumento di inclusione sociale nelle aree disagiate di Rio de Janeiro - Seconda fase

Centro di orientamento e formazione per giovani e donne a Salvador Bahia

Economia solidale speranza per il nord est brasiliano

Adotta un ritmo; dialogo tra città per l'inclusione sociale

SABOR NATURAL DO SERTÃO: appoggio alla commercializzazione dei prodotti

dell'agricoltura familiare nel semi-arido nordestino - Brasile

Promozione centri per favorire l'inserimento lavorativo di giovani e sviluppo movimento

cooperativo in Brasile - II anno

Il Rio della speranza - Progetto di sviluppo economico e sociale sostenibile dei territori

extraurbani del basso Rio Negro, come deterrente alla tragica emigrazione dei giovani verso

la città

Progetto di sostegno economico solidale alla cooperativa Spazio dei Sogni

Appoggio alla creazione di attività generatrici di reddito per gli agricoltori familiari delle comunità del litorale del Piauì

Centro di orientamento e formazione per giovani e donne a Salvador Bahia

Costruirsi un futuro. Progetto di promozione di inserimento sociale a favore di adolescenti e giovani in istituto di Belem, Stato del Parà

Il Rio della speranza - Progetto di sviluppo economico e sociale sostenibile dei territori

extraurbani del basso Rio Negro, come deterrente alla tragica emigrazione dei giovani verso

la città

Oficina de Rua

2008

Sviluppo del movimento cooperativo ed accesso al lavoro per giovani e donne, Brasile

PARA JOVENS

Lotta all'esclusione sociale e lavorativa dei giovani e delle donne di tre aree disagiate del municipio di Rio de Janeiro attraverso iniziative di orientamento al lavoro e promozione della microimprenditorialità

Il RIO della speranza

La costruzione collettiva del futuro: nuovi percorsi per l'imprenditoria solidale e il movimento

Poli di sviluppo agroeconomico per il rafforzamento dell'agricoltura familiare nella regione sud dello stato di Bahia. Una rete oltre i confini

Promozione dell'economia sociale attraverso il rafforzamento delle reti di sostegno

dell'agricoltura familiare e del Turismo rurale negli Stati del Piaù̀ e della Bahia

Valorizzazione, inserimento e promozione dell'impiego 
Rafforzamento dell'economia solidale e del cooperativismo in Brasile 2010

REdes de ECOnomia Solidaria - RECOS: Rafforzamento, ampliamento e moltiplicazione di reti di agroindustria solidale e di Turismo responsabile nei territori rurali degli Stati di Piauì, Maranhao, Bahia

Fonte: Elaborazione S. Grandi su database RER-ERVETcooperazionedecentrata, 2011.

Fino a questa fase l'istituzione regionale ha svolto un ruolo indiretto di coordinamento, programmazione e cofinanziamento a favore dell'animazione delle organizzazioni del territorio, ma dopo cinque anni questo fermento così come la congiuntura politica dell'elezione di Lula spinse ad una collaborazione strutturata tra la Regione Emilia-Romagna e la Repubblica federativa del Brasile. Nel 2004 alla luce dell'intensificarsi dei legami che in quegli anni si erano sviluppati tra le realtà territoriali emiliano-romagnole e quelle brasiliane (tra queste: università, città, organizzazioni sindacali, organizzazioni cooperative e comunità degli emigrati) la cooperazione divenne anche diretta. Fu firmato un accordo di collaborazione con un approccio basato sul principio di reciprocità. Gli ambiti di collaborazioni furono basati sull'approccio dello sviluppo locale, con particolare attenzione a:

- scambi di esperienze nel campo dell'economia solidale e delle imprese cooperative;

- promozione e qualificazione dei servizi a sostegno delle PMI;

- metodologie di pianificazione territoriale integrata ed organizzazione dei servizi pubblici locali;

- strumenti metodologici per l'integrazione delle politiche sociali con particolare riferimento ai territori;

- promozione culturale, turistica e formativa.

A quest'apertura seguirono varie iniziative progettuali sia indirette sia dirette con missioni reciproche di delegazioni istituzionali e tecniche, un accordo operativo annuale con il Sebrae Paranà per la collaborazione con la presenza continuativa di un funzionario della Regione a Curitiba (20082015), la sottoscrizione accordo di collaborazione al Governo dello Stato del Paranà e Regione EmiliaRomagna (2008-2012) con un addendum sul tema della collaborazione nel settore agroalimentare e la partecipazione attiva al programma Brasil Próximo che parzialmente ha capitalizzato l'esperienza e gli indirizzi della progettazione internazionale di quegli anni.

\section{IL PROGRAMMA BRASIL PRÓXIMO}

La proposta del programma Brasil Próximo è scaturita da un lungo percorso di collaborazione che le Regioni/Stati di entrambi i Paesi hanno realizzato fin dalla fine degli anni ottanta attraverso azioni di scambio informativo, istituzionale e tecnico, nonché grazie alla messa a disposizione di risorse finanziarie da parte del Ministero italiano degli Affari Esteri e della Cooperazione Internazionale, circa 4,7 milioni di euro, attraverso un meccanismo di programmazione negoziata e 
l'attivazione del Consiglio misto italo-brasiliano nel 2006 che vide la partecipazione anche delle regioni proponenti. Si trattava di fare un salto di qualità di coordinamento ed integrazione degli interventi per ridurre duplicazioni e dispersioni, svolgere sinergie con la cooperazione allo sviluppo internazionale italiana, di rendere maggiormente significativo l'impatto degli interventi promossi. Il punto di forza che si riteneva fondamentale era il costante coinvolgimento della controparte e degli altri attori locali brasiliani durante la fase di analisi territoriale, di identificazione delle azioni qui proposte nell'arco del triennio 2004-2007 di attuazione del progetto denominato "Percorso di Collaborazione per l'Implementazione di Politiche di Sviluppo Locale Integrato tra le Regioni delle Marche, Toscana, Umbria, Emilia-Romagna e Presidenza della Repubblica Federativa del Brasile" che le regioni proponenti e la Presidenza della Repubblica federativa del Brasile hanno cofinanziato nell'ambito dell'Accordo di Cooperazione Internazionale che è stato firmato a Roma l'1 luglio 2004 tra le Regioni Umbria, Marche, Toscana ed il Governo Federale Brasiliano cui ha successivamente aderito anche la Regione Emilia- Romagna. La Regione Liguria ha aderito al progetto a partire dal 2006.

Questo modo di lavorare ha garantito una governance plurale e un approccio bottom-up coerente con l'obiettivo del programma, ossia attivare nel medio periodo un network di supporto alle politiche, di opportunità e di interventi tesi ad "accompagnare processi endogeni di sviluppo locale capaci di intervenire sulle principali contraddizioni del paese" per "favorire il miglioramento delle condizioni di vita delle fasce deboli della società brasiliana", come recita il testo di presentazione del programma e della strategia d'intervento scritto nel 2008. In particolare l'obiettivo specifico era "mirato al rafforzamento delle politiche 'federative' brasiliane (Governo Federale, Stati e Municipi) rivolte a pianificare ed implementare interventi di sviluppo locale integrato e a sostenere i piccoli produttori attraverso la crescita delle microimprese, delle PMI e del cooperativismo" e i risultati attesi, per quanto ampi nella declaratoria, erano:

- sviluppare e consolidare una metodologia di intervento pubblico per lo sviluppo economico locale integrato capace di supportare il miglioramento delle politiche federative;

- sviluppare un nuovo approccio di politiche sociali basato sul patto territoriale quale strumento di concertazione e programmazione locale e sull'integrazione dei programmi federali, statali e municipali;

- concludere il processo di definizione del quadro normativo del cooperativismo e consolidare la politica di promozione di questo settore anche attraverso il supporto ad esperienze regionali ad alto valore dimostrativo nel contesto dello sviluppo locale, sia sociale che economico.

Per raggiungere questo obiettivo si è creato un insieme integrato di progetti che valorizzassero le esperienze di partenariato precedenti e le vocazioni, competenze e bisogni dei territori come schematizzato nella tabella 2 e negli schemi concettuali in figura 1 e 2 . 
Tabella 2: Articolazione dei progetti del Programma Brasil Próximo

\begin{tabular}{|c|c|c|c|}
\hline Titolo & Aree di Intervento & $\begin{array}{l}\text { Regione } \\
\text { Italiana }\end{array}$ & Partner brasiliani \\
\hline $\begin{array}{l}\text { sostegno allo } \\
\text { sviluppo } \\
\text { economico } \\
\text { integrato della } \\
\text { regione dell'Alto } \\
\text { Solimões- } \\
\text { Amazonas }\end{array}$ & $\begin{array}{l}\text { Stato di Amazonas, } \\
\text { regione dell'Alto } \\
\text { Solimões }\end{array}$ & $\begin{array}{l}\text { Regione Liguria, } \\
\text { attraverso enti } \\
\text { attuatori } \\
\text { selezionati }\end{array}$ & $\begin{array}{l}\text { Presidenza della Repubblica, SEPLAN } \\
\text { (Segreteria di stato alla pianificazione dello } \\
\text { Stato di Amazonas), Amazonastur (Agenzia } \\
\text { per lo sviluppo del turismo in Amazzonia), } \\
\text { Consorzio dei Comuni dell'Alto Solimoes, } \\
\text { Unità accademica "centro de estudos } \\
\text { superiores de Tabatinga" dell'Università dello } \\
\text { stato di Amazonas - UEA - corso di laurea in } \\
\text { turismo, Unità accademica "Instituto da } \\
\text { Natureza e Cultura di Benjamin Constant" } \\
\text { dell'Università Federale di Amazonas - UFAM }\end{array}$ \\
\hline $\begin{array}{l}\text { Valorizzazione } \\
\text { della filiera } \\
\text { dell'olio } \\
\text { extravergine di } \\
\text { oliva a Bagé, } \\
\text { Regione di } \\
\text { Campanha - RS }\end{array}$ & $\begin{array}{l}\text { Stato Rio Grande do Sul, } \\
\text { regione di Campanha, } \\
\text { Municipi di Bagé, Acegua, } \\
\text { Candiota, Hulha Negra } \\
\text { Chacapava do Sul, } \\
\text { Santana do Livramento }\end{array}$ & $\begin{array}{l}\text { Regione } \\
\text { Umbria, } \\
\text { attraverso } \\
\text { SviluppoUmbri } \\
\text { a }\end{array}$ & $\begin{array}{l}\text { Presidenza della Repubblica; Ministero dello } \\
\text { Sviluppo Agrario (MDA); Emater/RS; } \\
\text { Embrapa Clima Temperado Embrapa } \\
\text { CENARGEN, IFISUL, URCAMPA, UNIPAMPA, } \\
\text { UERGS e FEPAGRO }\end{array}$ \\
\hline $\begin{array}{l}\text { Creazione di una } \\
\text { rete di } \\
\text { strumenti a } \\
\text { sostegno delle } \\
\text { PMI della } \\
\text { Regione del } \\
\text { Centro Paulista } \\
\text { - SP }\end{array}$ & $\begin{array}{l}\text { Comuni di Araraquara, } \\
\text { São Carlos, Rio Claro, } \\
\text { Descalvado, Itirapina, } \\
\text { Riberão Bonito }\end{array}$ & $\begin{array}{l}\text { Regione } \\
\text { Umbria, } \\
\text { attraverso } \\
\text { SviluppoUmbri } \\
\text { a }\end{array}$ & $\begin{array}{l}\text { Presidenza della Repubblica; Ministero dello } \\
\text { sviluppo industriale e del commercio (MDIC); } \\
\text { Sebrae, Università UNESP, UNIARA, UFSCAR }\end{array}$ \\
\hline $\begin{array}{l}\text { Osservatorio sul } \\
\text { turismo della } \\
\text { regione della } \\
\text { Mantiqueira - SP }\end{array}$ & $\begin{array}{l}\text { Caxambu, Campos do } \\
\text { Jordão, Carmo de Minas, } \\
\text { Cristina, Itajubá, Maria da } \\
\text { Fé, Piranguinho, } \\
\text { Piranguçu; Santo Antônio } \\
\text { do Pinhal, Sapucaí Mirim, } \\
\text { São Lourenço, São Bento } \\
\text { do Sapucaí }\end{array}$ & $\begin{array}{l}\text { Regione } \\
\text { Toscana, } \\
\text { attraverso Ong } \\
\text { Cospe }\end{array}$ & $\begin{array}{l}\text { Caxambu, Campos do Jordão, Carmo de Minas, } \\
\text { Cristina, Itajubá, Maria da Fé, Piranguinho, } \\
\text { Piranguçu; Santo Antônio do Pinhal, Sapucaí } \\
\text { Mirim, São Lourenço, São Bento do Sapucaí. }\end{array}$ \\
\hline $\begin{array}{l}\text { Appoggio alla } \\
\text { costruzione del } \\
\text { quadro } \\
\text { normativo e } \\
\text { degli strumenti } \\
\text { operativi per il } \\
\text { cooperativismo } \\
\text { in Brasile }\end{array}$ & $\begin{array}{l}\text { Brasilia, Area } \\
\text { amazzonica, Piauí, } \\
\text { Paranà, cono Sud del } \\
\text { Brasile }\end{array}$ & $\begin{array}{l}\text { Regione Emilia- } \\
\text { Romagna, } \\
\text { attraverso enti } \\
\text { attuatori } \\
\text { selezionati }\end{array}$ & $\begin{array}{l}\text { Presidenza della Repubblica Brasiliana, } \\
\text { Ministero Politiche Sociali Brasile, Ministero } \\
\text { dell'educazione, Ministero del Lavoro, } \\
\text { Ministero dell'Agricoltura, Ministero dello } \\
\text { Sviluppo Agrario, Ministero dell'Ambiente. } \\
\text { Centrali cooperative brasiliane: OCB, Unisol, } \\
\text { Unicafes: Centro Ecologico, Ecocitrus, SEBRAE } \\
\text { Nazionale, Sebrae Paranà, Ocepar, } \\
\text { Organizzazione cooperative Stato del Paranà, } \\
\text { Codetec, Cooperativa Centrale di Ricerca } \\
\text { Agricola, Fundetec, FPTI - Fondazione parco } \\
\text { tecnologico Itaipu, Caciopar, Copacol, } \\
\text { Coopavel, C. Vale, Frimesa, Cooperativa } \\
\text { Agroindustriale, La Copercachaça, Ministero } \\
\text { Agricoltura Stato del Paranà, Se Scoop, } \\
\text { Segreteria Agricoltura e Attività Produttive } \\
\text { del Paraná -SEAB. Instituto Paranaense di } \\
\text { assistenza tecnica e sviluppo rurale EMATER, } \\
\text { Centrale di Rifornimento Ortofrutticolo del } \\
\text { Paraná - CEASA. Assessorato Comunale di }\end{array}$ \\
\hline
\end{tabular}




\begin{tabular}{|c|c|c|c|}
\hline & & & $\begin{array}{l}\text { Acquisti per Curitiba - SMAB, Cooperative e } \\
\text { produttori della zona interna del Pernambuco }\end{array}$ \\
\hline $\begin{array}{l}\text { Valorizzazione } \\
\text { turistica della } \\
\text { regione della } \\
\text { Serra das } \\
\text { Confusoes - } \\
\text { Piauí }\end{array}$ & $\begin{array}{l}\text { Municipalità di Caracol, } \\
\text { Jurema, Anisio De Abreu, } \\
\text { Tamboril Do Piauí, Canto } \\
\text { Do Buriti, Alvorada Do } \\
\text { Gurgueia, Cristino Castro, } \\
\text { Santa Luz, Guaribas, Bom } \\
\text { Jesus, Curimatá e } \\
\text { Redenção Do Gurgueia } \\
\end{array}$ & $\begin{array}{l}\text { Regione } \\
\text { Toscana con la } \\
\text { collaborazione } \\
\text { di COSPE e } \\
\text { Federparchi }\end{array}$ & $\begin{array}{l}\text { Governo Federale (Presidenza della } \\
\text { Repubblica e Ministero del Turismo), ICMBio } \\
\text { (Istituto Chico Mendes) }\end{array}$ \\
\hline $\begin{array}{l}\text { Sostegno alla } \\
\text { promozione dei } \\
\text { processi di } \\
\text { democrazia } \\
\text { partecipativa e } \\
\text { alle politiche } \\
\text { sociali nel } \\
\text { territorio della } \\
\text { Serra das } \\
\text { Confusoes } \\
\text { (Piaui) e nella } \\
\text { Baixada } \\
\text { Fluminense (Rio } \\
\text { de Janeiro) }\end{array}$ & $\begin{array}{l}\text { Stato di Rio de Janeiro, cd. } \\
\text { Baixada Fluminense, } \\
\text { Municipi di Nova Iguaçu, } \\
\text { Mesquita, Japerí, São Jõao } \\
\text { de Meriti, favela Santa } \\
\text { Marta e quartiere Campo } \\
\text { Grande della città di RdJ; } \\
\text { Stato del Piaui, regione } \\
\text { Serra das Confusões, } \\
\text { Municipi di Anísio de } \\
\text { Abreu, Canto do Buriti, } \\
\text { Caracol, Cristino Castro, } \\
\text { Guaribas, Jurema, Santa } \\
\text { Luz, Tamboril do Piauí, } \\
\text { Alvorada, Bom Jesus, } \\
\text { Curimatà e Redencao de } \\
\text { Gurguela }\end{array}$ & $\begin{array}{l}\text { Regione } \\
\text { Marche, } \\
\text { attraverso enti } \\
\text { attuatori } \\
\text { selezionati }\end{array}$ & $\begin{array}{l}\text { Presidenza della Repubblica; MDS Ministero } \\
\text { delle Politiche Sociali; Caixa Economica } \\
\text { Federal; Stato del Piaù; Municipi della Serra } \\
\text { das Confusões, Stato di Rio de Janeiro; } \\
\text { Municipi della Baixada Fluminense (municipi } \\
\text { di Nova Iguacu, Mesquita e Japeri); Istituto } \\
\text { Italiano di Cultura di RdJ; IBASE - Istituto } \\
\text { Brasilero de Analises Sociais e Economicas }\end{array}$ \\
\hline
\end{tabular}

Fonte: elaborazione documentazione di progetto e sito istituzionale www.brasilproximo.com, 2017. 
Figura 1: Mappa della gestione del Programma Brasil Próximo

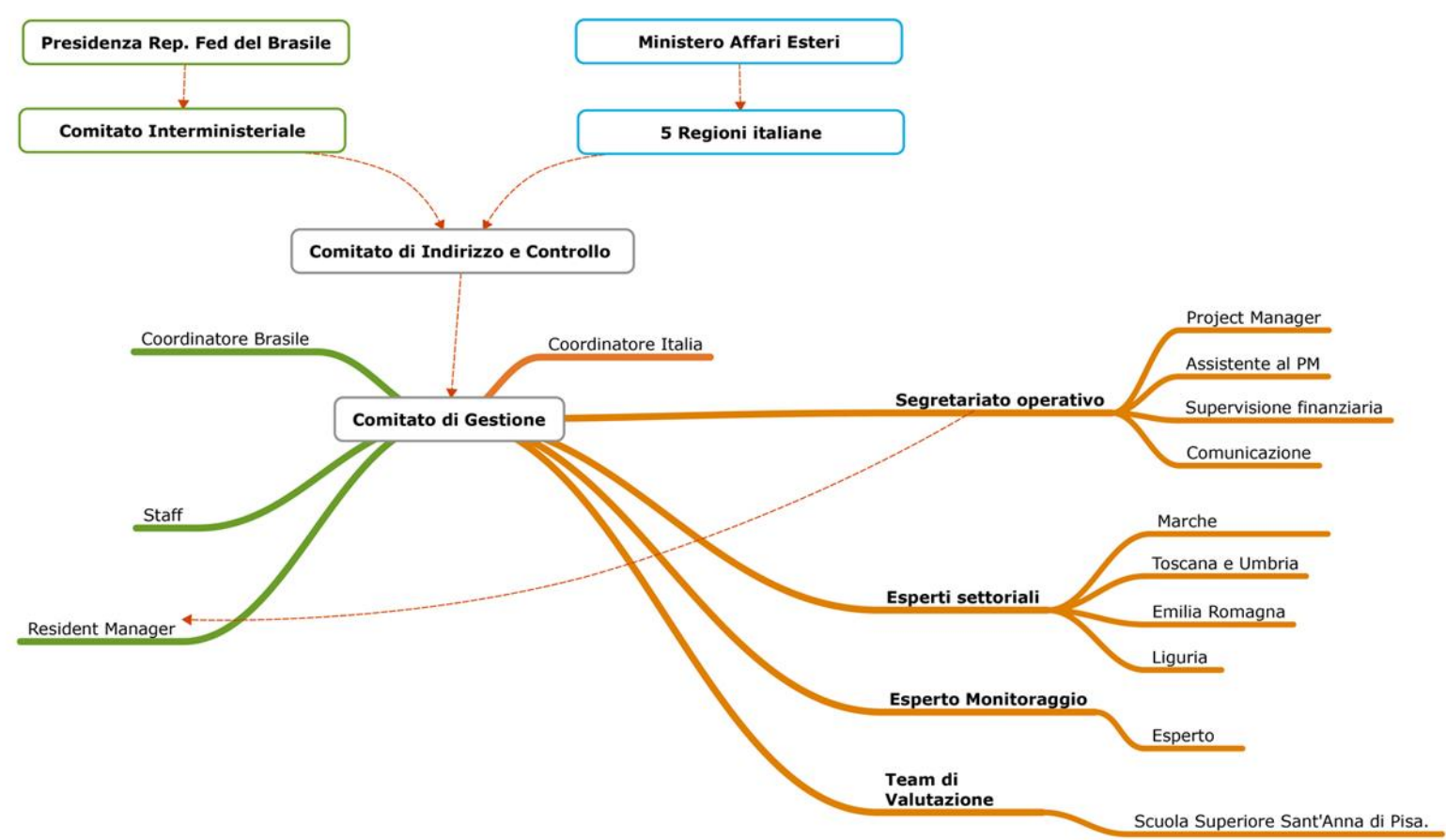

Fonte: sito istituzionale del programma www.brasilproximo.com, 2017

Figura 2: Schema concettuale dei progetti sul territorio del Programma Brasil Próximo

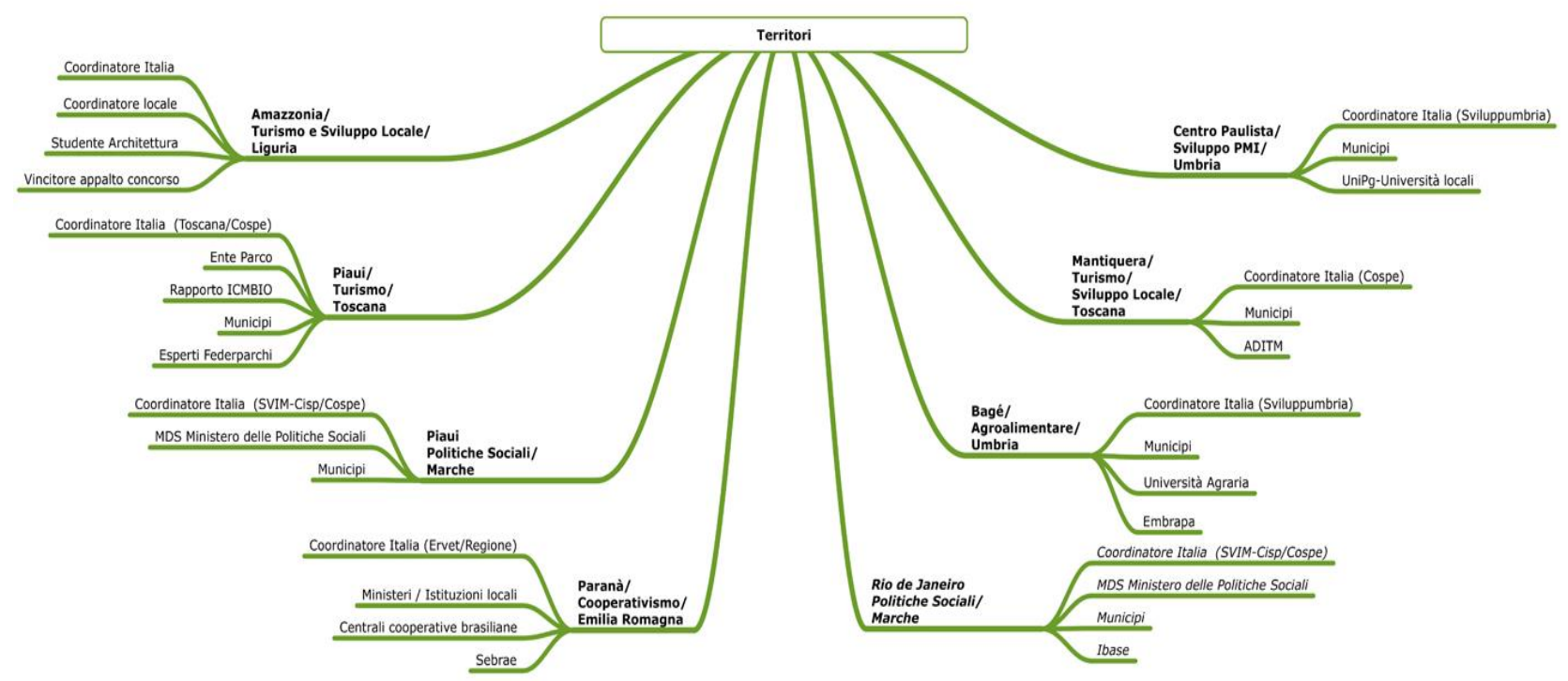

Fonte: sito istituzionale del programma www.brasilproximo.com, 2017. 


\section{IL RUOLO DELLA REGIONE EMILIA-ROMAGNA PER LA FILIERA AGROALIMENTARE DI QUALITÀ}

Nel processo di relazione di partenariato nell'ambito della cooperazione allo sviluppo si possono enucleare alcune fasi che si rileggono chiaramente nella storia della relazione EmiliaRomagna - Brasile. Dopo primi incontri esplorativi (università, sindacati, imprese) e missioni di delegazioni istituzionali, si passa prima al supporto finanziario a progetti di attori altri, poi all'impegno diretto di personale interno all'istituzione in un progetto. La relazione si consolida, in modo significativo, con la presenza costante di personale delle rispettive amministrazioni nella sede dell'altra. Così è avvenuto dal 2008 al 2015: la Regione Emilia-Romagna, grazie al progetto Brasil Próximo, ha avuto la possibilità di garantire una presenza costante di una sua funzionaria a Curitiba, presso il Sebrae del Paraná (Servizio di appoggio alla micro e piccola impresa), per realizzare le attività previste all'interno del programma e attivare ulteriori opportunità di collaborazione tra i rispettivi territori e settori di interesse. Questo ha permesso di potenziare sostanzialmente l'attività per i vantaggi di prossimità sociale-organizzativi e spill-over che ne conseguono. Tra le varie attività, che ha coordinato la funzionaria, grande comunanza di interesse è stata trovata nello sviluppo del settore agroalimentare portando ad alcuni risultati significativi in termini numerici. In particolare, i dati delle relazioni della Regione Emilia-Romagna evidenziano:

(a) attività volte al rafforzamento della filiera produttiva ortofrutta: 3.800 piccole proprietà della grande Curitiba sono state info-formate su temi quali tracciabilità, certificazione, qualità e controlli grazie allo scambio di informazione con tecnici specializzati e delegazioni in EmiliaRomagna o Paranà;

(b) attività di assistenza tecnica per la modernizzazione dei centri agroalimentari (CEASA) in Paranà focalizzati sul miglioramento della filiera, dal campo alla grande distribuzione, creando collaborazioni scientifiche e metodologiche con Università di Bologna - Facoltà di Agraria, il CAAB ed il sistema cooperativo di entrambe le parti, Legacoop, Confcooperative e AGCI;

(c) attività di assistenza tecnica per le imprese ed i consorzi agroalimentari sulle tecniche di produzione e logistica di campo;

(d) attività di formazione delle agenzie brasiliane di assistenza agli agricoltori sui temi della logistica e catena del freddo, tecnologie e meccanica agricola, sistema di gestione e rapporto con la grande distribuzione;

(e) attività di sviluppo nella realizzazione di tre edizioni della fiera Hortifruti Brasil Show8, la fiera del settore ortofrutta di Curitiba in collaborazione con la rispettiva esperienza emilianoromagnola, Macfrut, con rispettivi stand e incontri d'affari;

\footnotetext{
${ }^{8}$ Anni 2013, 2014 e 2015.
} 
(f) attività per la realizzazione di un modello per la creazione di un sistema di certificazione ética in Brasile tra il Ministero Agricoltura del Brasile, il Ministero dello Sviluppo Agricolo, il sistema di certificazione partecipativa Brasiliana e l'ICEA, consorzio italiano per la Certificazione Etica ed Ambientale;

(g) attività di promozione istituzionale dell'internazionalizzazione reciproca anche in occasione dell'Expo2015 che si è tenuto a Milano;

(h) attività di valorizzazione e collaborazione nel settore gourmet dei prodotti d'eccellenza, sia rafforzando la conoscenza in materia di certificazioni di indicazione geografica protetta (IGP) che le metodologie per la promozione delle tradizioni del territorio come eccellenze del territorio e la valorizzazione delle produzioni di alta qualità portando ad un accordo di collaborazione tra Casa Artusi e la scuola di formazione professionale Fecomercio - SENAC con l'idea di strutturare il polo formativo di gastronomia, per migliorare le capacità professionali brasiliane.

Per quanto non risulti direttamente solo riconducibile al settore agroalimentare ma anche al quadro dei fattori abilitanti al rafforzamento del settore cooperativo e imprenditoriale nelle sue varie declinazioni, in base alle documentazioni progettuali della Regione Emilia-Romagna analizzate, emergono rilevanti le collaborazioni Emilia-Romagna-Paranà-Brasile dedicate alla realizzazione di studi normativi comparati e di occasioni di rafforzamento formativo relativamente a garanzie al credito e al cooperativismo.

Nel primo caso, si è trattato soprattutto di analizzare in termini comparati i due casi, soprattutto con il focus sulle piccole e medie imprese, nonché l'organizzazione di eventi che mettessero in luce problemi e prospettive operative di garanzie per l'accesso al credito. In questo caso sono stati coinvolti diversi servizi della Regione Emilia-Romagna (cooperazione internazionale, internazionalizzazione e attività produttive), il sistema delle centrali cooperative regionali con il relativo sistema dei consorzi fidi - un modello piuttosto di successo per alcuni anni in Italia -, il Sebrae Nazionale e quello del Paranà nonché il Banco Central, Banco do Brasil e Caixa.

L'altra iniziativa avviata da tempo è la periodica formazione di quadri cooperativi del Paranà. Dal 2007 si realizzano ogni anno attività formative sia in Brasile che in Emilia-Romagna in collaborazione con il sistema cooperativo emiliano-romagnolo, l’Università di Bologna sede di Buenos Aires, ONG. Da alcuni anni questa attività non è supportata finanziariamente direttamente dal Sebrae Paranà e OCEPAR-Sistema delle cooperative Brasiliane. Al 2015, nell'ambito di questo schema, si contano oltre 150 dirigenti di cooperative creando poi economie di rete relazionali che si possono concretizzare nel tempo con contatti preferenziali, continui scambi informativi e contratti tra 
cooperative brasiliane ed emiliano-romagnole9. Sempre nell'ambito della cooperazione in materia di cooperativismo, è stato realizzato uno studio complesso tra Brasile e Italia con il coinvolgimento in Brasile di Senaes, MAPA-Ministero Agricoltura, Dieese, Denacoop, Presidenza della Repubblica, ed in Italia del sistema delle cooperative dell'Emilia Romagna per creare un osservatorio sulle cooperative in Brasile ispirato alla realtà dell'Emilia-Romagna, compreso il centro di documentazione su cooperazione ed economia sociale di Bologna.

\section{CONCLUSIONI}

Il caso in esame mette in evidenza i diversi volti della cooperazione decentrata e le sue declinazioni. Innanzitutto emerge il il significato politico, è un impegno per la pace e la solidarietà che coinvolge la cittadinanza ed i suoi rappresentanti. Tra i vantaggi della cooperazione decentrata si riconosce una migliore capacità di mobilitare le strutture sociali ed economiche presenti sul territorio creando opportunità di partenariato con strutture omologhe dei paesi terzi e favorendo occasioni di democrazia partecipativa. Quando i vantaggi economici tra le realtà sono più forti e il baricentro si sposta in una logica di post-aid, come nel caso della relazione Italia-Brasile, la cooperazione attiva significative spirali di cooperazione anche con relazioni economiche e d'affari, con l'idea che "commercio internazionale e sviluppo" siano le chiavi per la crescita di entrambe le parti, portando vantaggi congiunti, oltre che per compensare il venir meno di fondi in forma di dono. Diventa inoltre una via importante di collaborazione in una logica di localizzazione dello sviluppo sostenibile (UNDP, 2012) nell'attuazione dell'Agenda 2030.

D’altra parte l'approccio della cooperazione decentrata presenta molti limiti per l'effetto della frammentazione degli interventi, della scarsa "massa critica" dei progetti, della mancanza di un quadro di riferimento nazionale ed internazionale, della debolezza dei rapporti di sussidiarietà verticale ed orizzontale, della scarsità dei finanziamenti per lo sviluppo e dell'insufficiente attenzione al rafforzamento istituzionale e alla democratizzazione nei paesi in via di sviluppo.

Va comunque detto che non si configura come sostitutiva della cooperazione portata avanti dai soggetti tradizionali (Governi e Organismi Internazionali, ONG Internazionali) ma piuttosto come aggiuntiva svolgendo un ruolo importante perché coinvolge soggetti altrimenti esclusi e apporta ulteriori risorse.

La tipologia di intervento è basata piuttosto sull'assistenza tecnica alle omologhe amministrazioni locali, agli enti pubblici e privati che gestiscono servizi di base per i cittadini (ospedali, scuole, ecc.) e alle comunità locali in generale per individuare e soddisfare i bisogni espressi.

\footnotetext{
${ }^{9}$ L'azione ha anche una dimensione più ampia e molte attività sono state svolte a Sao Bernardo do Campo (San Paolo) insieme all'Università metodista di Sao Paolo, UNISOL (Centrale delle cooperative Brasiliane, nate da imprese recuperate), il consorzio comuni dell'ABC Paulista, attraverso la realizzazione di corsi per addetti del settore cooperativo e per amministratori pubblici sul tema gestione cooperative e adeguamento normativo.
} 
Diventa, inoltre, per il sistema locale della regione sviluppata un'importante occasione di scambio culturale, di azione di aggregazione e discussione. È pur vero che se per alcuni paesi la cooperazione decentrata, intesa in senso statistico come "gli aiuti finanziati da enti pubblici e privati ed erogati attraverso le autorità locali, le reti e altri attori locali", rappresenti solo in alcuni Paesi una quota significativa degli aiuti allo sviluppo e che spesso questa definizione intenda quindi porre la cooperazione in un alveo definitorio riconducibile alle categorie OCSE - DAC, il ruolo delle Regioni e Città italiane può essere considerato piuttosto marginale e, soprattutto, in declino dopo la crisi, le riforme e quasi riforme del sistema amministrativo italiano.

D’altra parte, l'esperienza della Regione Emilia-Romagna, così come per le Regioni Toscana, Piemonte, Sardegna, Umbria, Marche, Molise e la Provincia Autonoma di Trento dimostrano che non può essere considerato un fenomeno episodico e marginale: la maggior parte delle Amministrazioni Comunali, Provinciali e la Regione hanno attività di relazione e progettazione internazionale che si palesa nei diversi modi della cooperazione decentrata o almeno di azione sul territorio di promozione dell'educazione, della sensibilizzazione e della partecipazione di tutti i cittadini alla solidarietà internazionale, alla cooperazione internazionale e allo sviluppo sostenibile.

Quindi, pur assumendo un ruolo marginale in termini finanziari ma non si può concludere lo stesso ragionando in termini di efficacia ed influenza nelle policy per lo sviluppo locale. La cooperazione decentrata è un meccanismo propulsivo per creare effetti a catena virtuosi nei luoghi.

Tuttavia, spesso questo fenomeno si perde di vista e non riesce ad essere riconosciuto. Si osserva una sorta di dissolvimento. Nel passaggio da istituzione ad istituzione, da persona a persona, si disperde la conoscenza dell'origine del fenomeno di cooperazione, si alimenta e si nutre di informazioni che vengono ricomposte nei vari luoghi e riattribuite nella società e nelle sue economie, dissolvendosi e non ritrovandosi più. Per il policy maker che necessita conferme sull'impatto delle politiche, sull'efficacia degli aiuti ed incentivi questo diventa un dilemma, un fallimento: ma lo è veramente o è appunto la realizzazione piena di un processo di co-sviluppo sostenibile tra pari ed endogeneizzato? O come dirlo à la Vallega (1995, pp. 79-80), i sistemi locali reagiscono alla relazione di cooperazione trasformandola in un progetto a cui si riconosce un valore identitario attraverso un processo autopoietico.

\section{REFERÊNCIAS}

AA.VV. (2008), "BRASIL PROXIMO.Cinque Regioni Italiane per lo Sviluppo Locale Integrato in Brasile", Testo del Progetto proposto da Regioni Umbria, Marche, Toscana, Emilia-Romagna, Liguria.
BACILLIERI C. (2006), La Consulta degli emiliano-romagnoli nel mondo: un patrimonio per l'intera Regione, inLe Istituzioni del Federalismo. Regione e Governo Locale. Bimestrale di studi giuridici e 
politici della Regione Emilia-Romagna, anno XXVII, Vol. 3, Supplemento.

BERTOCCIN M., Dansero E., Loda M., Pase A., Bini V., Burini F., Cerreti C., Gamberoni E., Grandi S., Isemberg T., Sommella R., Putilli M. (2016), Lasciarsi provocare dal Mondo. Geografia e cooperazione allo sviluppo: una relazione antica, un dibattito attuale, in Rivista Geografica Italiana, Vol. 124, Fasc. 3, Settembre 2016, pp. 347-358 - ISSN 00356637.

BIGNANTE E., Dansero E., Loda M. (2015), Geografia e cooperazione allo sviluppo: prospettive e agende di ricerca, in Geotema, 19, n. 48, pp. 5-24.

BIGNANTE E., Dansero E., Scarpocchi C. (2008), Sviluppo Locale e cooperazione internazionale. Una proposta teoricometodologica, in Bignante E., Dansero E., Scarpocchi C. (a cura di), Geografia e cooperazione allo sviluppo, Franco Angeli, Milano.

BIGNANTE E., Dansero E., Scarpocchi C., (2008), Geografia e cooperazione allo sviluppo. Temi e prospettive per un approccio territoriale. Utet, Torino.

BINI V. (2016), La cooperazione allo sviluppo in Africa. Teorie, politiche, pratiche, Mimesis Edizioni (Milano-Udine).

BOGGIO F., Dematteis G. (2002), Geografia dello sviluppo. Diversità e disuguaglianze nel rapporto Nord-Sud, Utet, Torino.

BOGGIO F., Dematteis G., Memoli M. (a cura di), (2008), Geografia dello sviluppo. Spazi, economie e culture tra ventesimo secolo e terzo millennio, Utet Università, Torino.

BONAGLIA F., De Luca V. (2006), La cooperazione internazionale allo sviluppo, Il Mulino, Bologna.

Commissione Europea, (2008), Comunicazione della Commissione al Consiglio, al Parlamento europeo, al Comitato economico e sociale europeo e al Comitato delle regioni $L E$ AUTORITÀ LOCALI: ATTORI DI SVILUPPO, Bruxelles, 8.10.2008 COM(2008) 626 definitivo - http://eurlex.europa.eu/LexUriServ/LexUriServ.do?uri $=$ COM:2008:0626:FIN:IT:PDF

DANSERO E. (2008), Geografia e cooperazione allo sviluppo. Prospettive di ricerca, in Bignante E., Dansero E., Scarpocchi C. (a cura di), Geografia e cooperazione allo sviluppo.
Temi e prospettive per un approccio territoriale, Franco Angeli, Milano.

DANSERO E., Scarpocchi C. (2014), Local development in international cooperation: issues, approaches and perspectives for a territorialisation of development policies, in JUNCO - Journal of Universities and international development Cooperation, Anno I, Vol. 1.

EASTERLY W.(2002), The elusive quest for growth, MIT Press, Cambridge, USA.

Fanini D., Preus R. (2001), Progetti di Emergenza e ricostruzione internazionale 1996-2000, Regione Emilia-Romagna, Bologna.

CCRE (2017), Cos'è il gemellaggio? Una breve panoramica http://www.twinning.org/it/page/unabreve-panoramica\#.WV_abITyjIU

Grandi S. (a cura di) (2013), Sviluppo, geografia e cooperazione internazionale. Teorie, politiche e mappamondi, Editrice La Mandragora, Imola.

GRANDI S.(2010), Geografia dello Sviluppo e dela cooperazione internazionale, Dipason Editore, Rimini.

GRANDI S., Curiazi R. (2013), La dimensione globale e locale dello sviluppo e le sue critiche, in Grandi S. (a cura di), Sviluppo, geografia e cooperazione internazionale. Teorie, politiche e mappamondi, Editrice La Mandragora, Imola.

MAECI-DGCS (2002), Linee di indirizzo e modalità di attuazione della collaborazione della Dgcs con le Regioni e gli Enti locali, Ministero degli Affari Esteri Italiano, CITTà? NGANJE F.(2015), Decentralized Cooperation and the New Development Cooperation Agenda: What Role for the UN?, United Nations University, New York.

OICS (2005), La cooperazione allo sviluppo. Il ruolo dela cooperazione decentrata, http://www.oics.it/datianalisiricerche.

PARESCHI S., BREVEGLIERI S. (2013), “Unisol, un nuovo modello cooperativo per il Brasile (prima parte)" Rapporto NEXUS http://www.inchiestaonline.it/le-sfide-delbrasile/nexus-cgil-emilia-romagna-unisol-unnuovo-modello-cooperativo-per-il-brasileprima-parte/

Platforma(2013), Five years of Action!, Platforma, Bruxelles. 
Sister Cities International (2006), Peace Through People: 50 Years of Global Citizenship, Butler Books, Kentucky and Canada http://www.sister-cities.org/mission-andhistory

UNDP (2012), "Localizing Sustainable Human Development: Consideration for Post-2015 Global Development Agenda", Policy Brief (United Nations Development Programme: New York).

UN-Habitat e UTO/FMCU (2001), "City-to-City Cooperation: Issues Arising from Experience. An Interim Report prepared as a contribution to discussions on decentralised cooperation at the IULA/UTO Unity Congress, Rio de Janeiro, 3-6 May 2001", World Associations of Cities and Local Authorities Coordination, Nairobi. UNIDO (2010), Cluster development for propoor growth: the UNIDO approach, Business, Investment And Technology Services Branch Technical paper series, UNIDO, Vienna. VALLEGA A. (1995), La Regione, sistema territoriale sostenibile, Mursia, Milano.

VANUCCI C. (2007), Gemellaggio: opportunità d'incontro tra culture, Tesi di Laurea, Università di Modena e Reggio Emilia. 